Proceedings

\title{
Design of a Wind Sensor Based on Cylinder Diametrical Pressure Differences for Boundary Layer Meteorological Observation ${ }^{+}$
}

\author{
Zhan Zhao ${ }^{1,2, *}$, Yichen Pan ${ }^{1,2}$, Rongjian Zhao ${ }^{1,2}$, Lidong Du ${ }^{1}$, Zhen Fang ${ }^{1,2}$, Hong $\mathrm{Wu}^{3}$ and \\ Xianghua Niu ${ }^{4}$ \\ 1 State Key Laboratory of TransducerTechnology, Institute of Electronics, Chinese Academy of Sciences, \\ Beijing 100190, China; panyichen.ucas@foxmail.com (Y.P.); rongjian8780@163.com (R.Z.); \\ lddu@mail.ie.ac.cn (L.D.); zfang@mail.ie.ac.cn (Z.F.) \\ 2 University of Chinese Academy of Sciences, Beijing 100049, China \\ 3 Key Laboratory of Transportation Meteorology, China Meteorological Administration, Nanjing 210008, \\ China; wu58283@163.com \\ 4 Beijing Institute of Applied Meteorology, Beijing 100029, China; nxh0322@126.com \\ * Correspondence: zhaozhan@mail.ie.ac.cn; Tel.: +86-10-58887186 \\ + Presented at the Eurosensors 2018 Conference, Graz, Austria, 9-12 September 2018. \\ Published: 10 December 2018
}

\begin{abstract}
Wind speed and direction are important parameters in meteorological observation. Solid wind sensor has needed with small Quadcopter for boundary layer meteorological observation. In this paper the principle of a cylindrical two-dimensional wind sensor is reported by analyzing the data from wind tunnel experiments. A model is proposed to describe the distribution of the pressure difference across a diameter of a cylinder and the wind sensor is fabricated with MEMS differential pressure sensors. In wind tunnel tests in range of $1 \sim 40 \mathrm{~m} / \mathrm{s}$, the relative speed measuring errors and the direction measuring errors of the prototype are no more than $\pm(0.2+0.03 \mathrm{~V}) \mathrm{m} / \mathrm{s}$ and $5^{\circ}$ respectively.
\end{abstract}

Keywords: two-dimensional wind sensor; wind speed and direction; pressure difference; meteorological observation

\section{Introduction}

Boundary layer meteorological observation is a development direction in the field of meteorological monitoring, which could determine various meteorological elements in the lower atmosphere and vertical distribution of pollutants and their change regularity. At present, the meteorological tower is still as a kind of meteorological observation facilities. With the quadcopter development, it become increasingly common, capable, and affordable, their utility as a mobile sensor platform continues to grow [1,2].

Wind direction and speed are the important parameters in meteorological observation, while the traditional wind sensor is not suitable to apply to the quadcopter for its large size and weight. Fortunately, MEMS technology pushed the development of the wind sensor, and made the devices with small size and low cost possible [3-5]. Even though that the thermal and mechanical sensitive elements of wind sensor must be direct contact with fluidic air, which easy be polluted and damaged in meteorological application [6].

It is worth to mention Bruschi P's works in this paper, which is based on both ends of the diameter of the cylinder pressure difference to measure the two-dimensional velocity vector, design structure into flow branch pipe to the pressure, and then use principle MEMS thermal flow sensor to measure the flow, and calculate the wind speed and direction [7]. Literature [8] 
completed the prototype of cylindrical two-dimensional wind sensor, wind speed measurements and standards of the anemometer and shown reveal consistency, the wind measurement error is no more than $8^{\circ}$. However all these wind sensors have some defects and cannot meet the need for boundary layer meteorological observation.

In this paper the principle of the wind sensor was introduced based on the cylinder diametrical pressure differences. The mathematical model was established and based on that the wind sensor was fabricated, in which four differential pressure sensor used to measure the pressure instead of tow flow sensors. It has advantage of small size and high accuracy in measurement of wind velocity and direction, and also has higher protection ability of dust and rain than based on MEMS flow sensors.

\section{Modeling}

Cylindrical two-dimensional anemometer research, involving fluid mechanics problem of flow around a cylinder, which are commonly used pressure coefficient $C p$ to describe cylindrical surface pressure distribution, its definition [9] is:

$$
C_{p}=\frac{p-p_{\infty}}{\frac{1}{2} \rho U_{\infty}^{2}}
$$

In which $p$ is the object surface pressure, $\rho, p \infty$ and $U_{\infty}$ are infinity fluid density, static pressure and flow rate separately. Ideally, the cylindrical surface point $X$, as shown in Figure 1, the pressure efficiency is:

$$
C p=1-4 \sin ^{2} \theta
$$

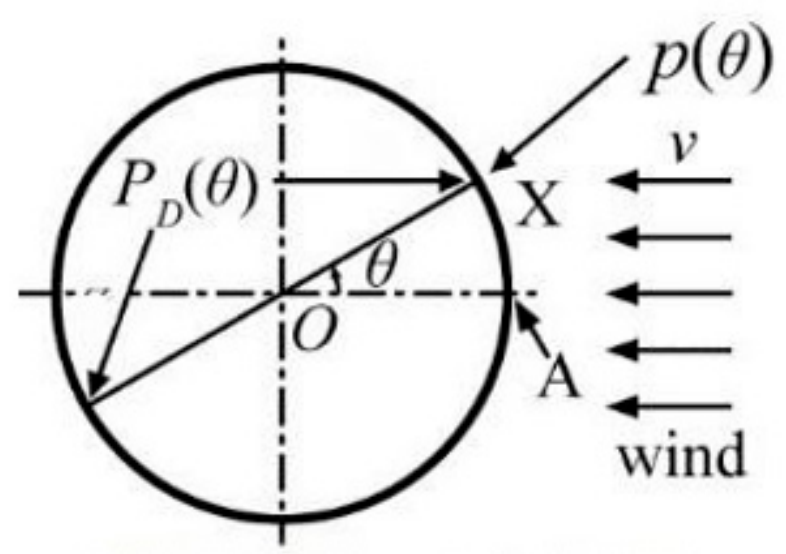

\section{A Front stationary $O$. Center}

Figure 1. The pressure and pressure difference of a cylinder.

The formula (2) shows that the pressures between the ends of a diameter in the column are equal. Actually the fluid viscosity can cause the change of flow state so that the cylindrical surface pressure distribution has obvious difference with the ideal case. Using fluid Reynolds number to determine the flow state, the definition is as following [7]

$$
\mathrm{Re}=\frac{\rho V D}{\mu}
$$

where $\rho, V$ and $\mu$ are fluid density, velocity, and dynamic viscosity coefficient respectively. $D$ is the diameter of the cylinder. 
There is a choked cylindrical pressure difference usually between the two ends of a diameter. As shown in Figure 1, here defines $P_{D}(\theta)$ as the pressure difference on both ends of the diameter, or difference in the center $\mathrm{O}$ as the positive side and negative side pressure, including $\theta$ as the diameter of the azimuth Angle, namely the diameter is the end and the stagnation point A counterclockwise angle range. Define the pressure differential coefficient of $C_{P D}(\theta)$ as follows:

$$
C_{P D}(\theta)=\frac{P_{D}(\theta)}{\frac{1}{2} \rho U_{\infty}^{2}}
$$

\section{Wind Tunnel Experiments}

We fabricated a wind speed sensor for the experiment using a cylindrical with $26 \mathrm{~mm}$ in diameter. There is a hole with diameter of $1 \mathrm{~mm}$ on both ends of the cylindrical diameter for sensing the pressure. Pressure hole inside through the pipe connected to the differential pressure sensors to measure the pressure difference on both ends of the diameter.

The pressure difference coefficient values measured between choked diameters on both ends are shown in Figure 2.

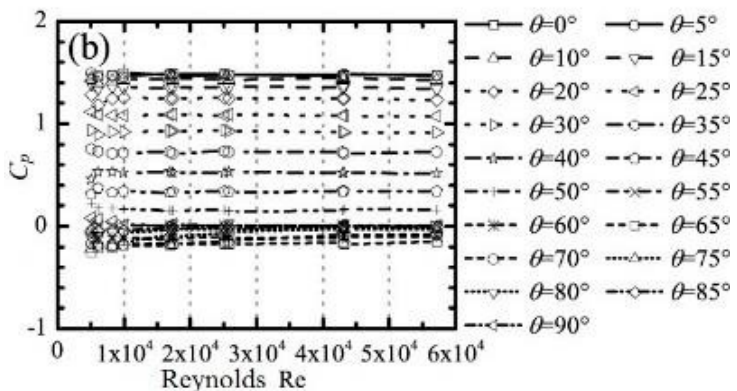

(a)

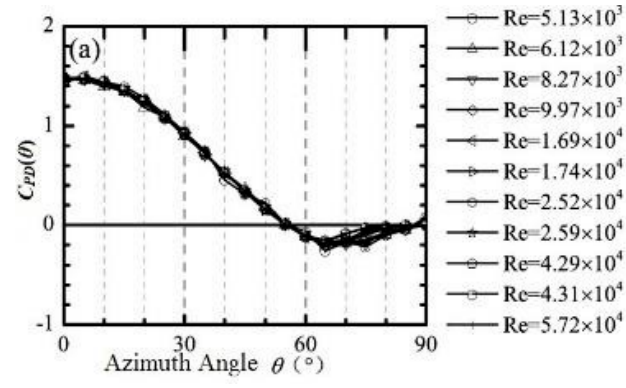

(b)

Figure 2. The measured pressure difference coefficients to (a) Reynolds numbers and (b) the azimuth angles.

Figure 2a is a kind of representation of the distribution of the pressure difference coefficient, Figure $2 \mathrm{~b}$ shows the $C p_{D}(\theta)$ with non-monotonicity, it is monotonous about azimuth distribution within the scope of $0^{\circ} \sim 60^{\circ}$, but near $65^{\circ}$ the value is zero. Therefore, wind velocity and direction are need to measure along with four diameter in time in the range of $0^{\circ}$ to $45^{\circ}$. Therefore, the mathematical model of the pressure difference on both ends is as following:

$$
C_{P D}(\theta)=A \cos ^{2} \theta+\mathrm{B}, \theta \in\left[0^{\circ}, 45^{\circ}\right]
$$

\section{Prototype of Wind Sensor and Results}

The prototype of wind sensor consists of a $30 \mathrm{~mm}$ diameter, $80 \mathrm{~mm}$ cylindrical probe with four MEMS differential pressure sensor, a temperature sensor, MCU and peripheral circuit Figure 3. Eight pressure holes equally arranged in a cylindrical, spacing between $45^{\circ}$. The results shown the wind sensor has excellent properties as Figures 4 and 5.

The two-dimensional wind sensor was tested in a wind tunnel test. Each wind speed point, at $360^{\circ}$ with $10^{\circ}$ step scanning range of the wind.

Figures 4 and 5 show the relationship between the given tunnel wind speed and measured speed by the designed wind sensor for each wind speed. The relative error is not more than $\pm 5 \%$. When the wind speed is more than $5 \mathrm{~m} / \mathrm{s}$. The wind direction error is not more than $\pm 5^{\circ}$. We 
have tested the wind sensor in a quadcopter and compared with cup wind sensor in the tower. The results are shown in Figure 6.

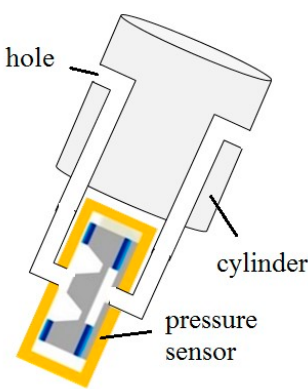

(a)

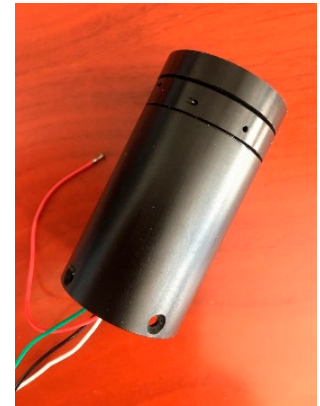

(b)

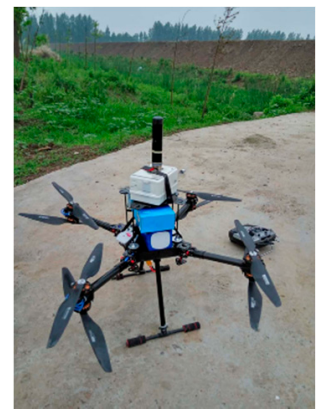

(c)

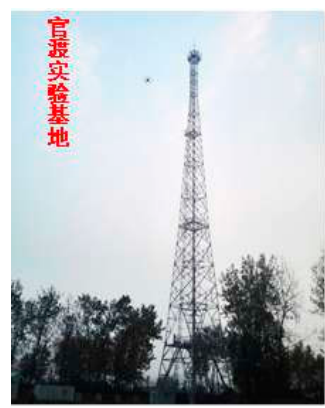

(d)

Figure 3. The prototype of wind sensor and quadcopter meteorological application. (a) The principle of measurement; (b) prototype of the wind sensor; (c) application on a quadcopter; (d) tested with meteorological tower.

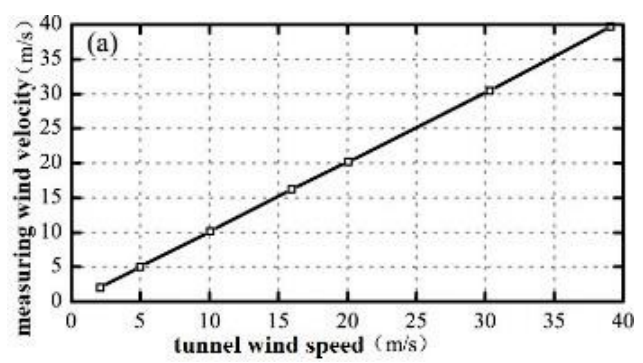

(a)

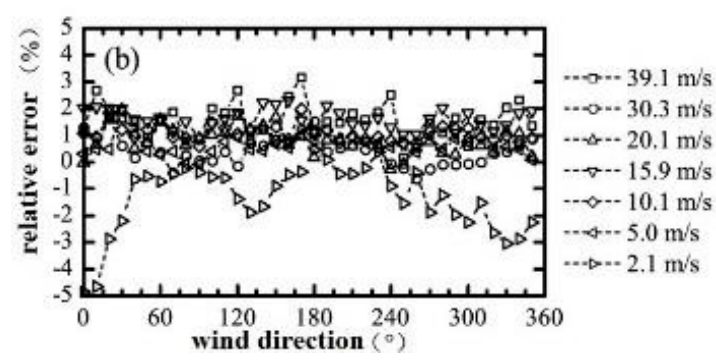

(b)

Figure 4. The wind speed test results, (a) linear wind speeds relationship and (b) the relative errors.
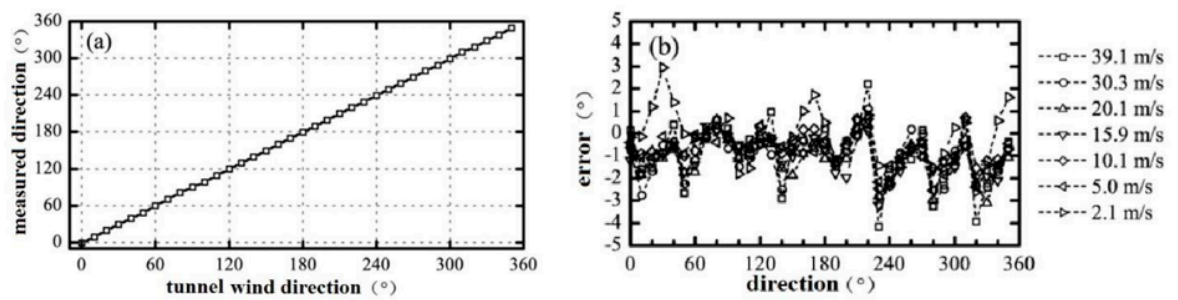

Figure 5. The wind direction test results, (a) The linear wind direction relationship and (b) the relative errors.
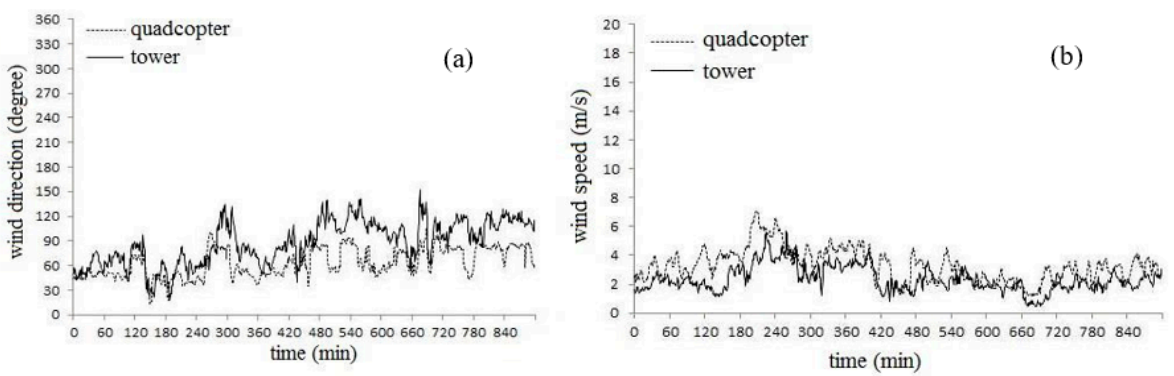

Figure 6. Boundary layer meteorological observation test compared in the height $70 \mathrm{~m}$ between our wind sensor on a quadcopter and traditional wind sensor in tower, (a) wind direction and (b) wind speed. 


\section{Conclusions}

In this paper a two-dimensional wind sensor measuring principle was proposed. A mathematical model is established to describe the choke pressure difference distribution on bot $h$ ends of the cylinder diameter. According to the principle, a prototype of two-dimensional wind sensor based on MEMS differential pressure sensor was fabricated, which has the properties of small size and high accuracy. Prototypes have been used to the quadcopter for boundary layer meteorologicalobservationapplication.

Acknowledgments: This work was supported by Key Project of Beijing Natural Science Foundation (Grant Z16002) and Special Scientific Research Fund of Meteorological Public Welfare Profession of China (Grant GYHY201006044).

Conflicts of Interest: The authors declare no conflict of interest.

\section{References}

1. Bruschi, P.; Piotto, M.; Dell'Agnello, F.; Ware, J.; Roy, N. Wind speed and direction detection by means of solid-state anemometers embedded on small quadcopters. Procedia Eng. 2016, 168, 802-805.

2 Piotto, M.; Pennelli, G.; Bruschi, P. Fabrication and characterization of a directional anemometer based on a single chip MEMS flow sensor. Microelectron. Eng. 2011, 88, 2214-2217.

3. Kuo, J.T.K.; Yu, L.; Meng, E. Micromachined Thermal Flow Sensors - A Review. Micromachines 2012, 3, 550573.

4 Du, L.; Zhao, Z.; Pang, C.; Fang, Z. Drag force micro solid state silicon plate wind velocity sensor. Sens. Actuators A Phys. 2009, 151, 35-41.

5. Zhu, Y.; Chen, B.; Qin, M.;Huang, J.Q.; Huang, Q.A. Development of a self-packaged 2D MEMS thermal wind sensor for low power applications. J. Micromech. Microeng. 2015, 25,1-14

6 Dong, Z.; Chen, J.; Qin, Y.; Qin, M.; Huang, Q.A. Fabrication of a Micromachined Two-Dimensional Wind Sensor by Au-Au Wafer Bonding Technology. J. Microelectromech. Syst. 2012, 21, 467-475.

7. Bruschi, P.; Dei, M.; Piotto, M. A low-power 2-D wind sensor based on integrated flow meters. IEEE Sens. J. 2009, 9, 1688-1696.

8. Liu, C.; Du, L.; Zhao, Z. A directional cylindrical anemometer with four sets of differential pressure sensors. Rev. Sci. Instrum. 2016, 87, 035105.

C 2018 by the authors. Licensee MDPI, Basel, Switzerland. This article is an open access article distributed under the terms and conditions of the Creative Commons Attribution (CC BY) license (http://creativecommons.org/licenses/by/4.0/). 